\title{
Optimal Vehicle Dispatching for Ride-sharing Platforms via Dynamic Pricing*
}

\author{
Mengjing Chen \\ IIIS, Tsinghua University \\ Haidian District, Beijing, China \\ ccchmj@qq.com \\ Pingzhong Tang \\ IIIS, Tsinghua University \\ Haidian District, Beijing, China \\ kenshinping@gmail.com
}

\author{
Weiran Shen \\ IIIS, Tsinghua University \\ Haidian District, Beijing, China \\ emersonswr@gmail.com \\ Song Zuo \\ IIIS, Tsinghua University \\ Haidian District, Beijing, China \\ songzuo.z@gmail.com
}

\begin{abstract}
Over the past few years, ride-sharing has been proven to be an effective way to relieve urban traffic congestion, as evidenced by several emerging ride-sharing platforms such as Uber and Didi. A key economic problem for these platforms is to design a revenueoptimal (or welfare-optimal) pricing scheme and a corresponding vehicle dispatching policy that incorporates geographic information, and more importantly, dynamic supply and demand. In this paper, we aim to solve this problem by introducing a unified model that takes into account both travel time and driver redirection. We tackle the non-convexity problem using the "ironing" technique and formulate the optimization problem as a Markov decision process (MDP), where the states are the driver distributions and the decision variables are the prices. Our main finding is to give an efficient algorithm that computes the exact revenue (or welfare) optimal randomized pricing schemes. We characterize the optimal solutions of the MDP by primal-dual analysis of a convex program. We also conduct empirical analysis of our solution with real data of a major ride-sharing platform and show its significant advantages over fixed pricing schemes as well as those prevalent surge-based pricing schemes.
\end{abstract}

\section{CCS CONCEPTS}

- Theory of computation $\rightarrow$ Computational pricing and auctions; Algorithmic game theory and mechanism design;

\section{KEYWORDS}

Ride-sharing; dynamic pricing; vehicle dispatching

\section{ACM Reference Format:}

Mengjing Chen, Weiran Shen, Pingzhong Tang, and Song Zuo. 2018. Optimal Vehicle Dispatching for Ride-sharing Platforms via Dynamic Pricing. In WWW '18 Companion: The 2018 Web Conference Companion, April 23-27,

"We thank the anonymous reviewers for their helpful comments. This paper was supported in part by the National Natural Science Foundation of China Grant 61561146398 , a China Youth 1000-talent program and an Alibaba Innovative Research program.

This paper is published under the Creative Commons Attribution 4.0 International (CC BY 4.0) license. Authors reserve their rights to disseminate the work on their personal and corporate Web sites with the appropriate attribution.

WWW'18 Companion, April 23-27, 2018, Lyon, France

() 2018 IW3C2 (International World Wide Web Conference Committee), published under Creative Commons CC BY 4.0 License.

ACM ISBN 978-1-4503-5640-4/18/04

https://doi.org/10.1145/3184558.3186924
2018, Lyon, France. ACM, New York, NY, USA, 2 pages. https://doi.org/10. $1145 / 3184558.3186924$

\section{INTRODUCTION}

The emerging applications of shared mobility, such as ride-sharing, bike-sharing, and car-sharing, have been proven to be an effective way to utilize transportation resources and to improve social welfare [7]. Over the past few years, intensive researches have been done on topics related to the economic aspects of sharing mobility $[8,12,15]$.

Despite of existing literatures, the problems of how to design revenue optimal (or welfare optimal) prices and dispatching schemes in these environments, to the best of our knowledge, are still not well understood. There are at least two challenges when one wants to tackle this problem. First of all, due to the nature of transportation, the pricing and dispatching scheme must be geographically dependent. Secondly, the pricing and dispatching scheme should take into consideration the fact that the supply and the demand in these environments may change over time. As a result, it may be difficult to compute, or even to represent a price and dispatching scheme for such complex environments.

Traditional pricing and dispatching schemes for taxis $[10,11,13]$ and airplanes $[14,16]$ do not capture the dynamic nature of the environments under consideration in this paper: (i) the dispatching system for these shared vehicles can make use of dynamic pricing to further boost revenue, while taxi fees calculated by traditional schemes are based on a fixed function of traveling distance and time; (ii) the prices of flight tickets are sold via relatively long booking periods, in contrast, the customers of shared vehicles make their decisions rather immediately.

\subsection{Model}

In this paper, we propose a graph based model to analyze the vehicle dispatching and pricing problem mentioned above. In the graph, each node refers to a region in the city and each edge refers to a possible trip that includes a pair of origin and destination as well as a cost associated with the trip on this edge.

The design problem is, for the platform, to set a price for each edge at each time step as well as the vehicle dispatches induced by the supply and demand at the origin of that edge under this price 
at this time. The objective of the platform can either be its revenue or the social welfare or any convex combination between them.

\subsection{Techniques}

Our model naturally induces a Markov Decision Process (MDP) with the driver distributions on each node as states, the throughputs along each edge as actions, and the objective (e.g., revenue or welfare) as the rewards. While it is in general PSPACE-hard to solve MDPs, we come up with an efficient (polytime) algorithm that finds the exact optimal solution.

Our first key step that makes the MDP trackable is to formulate the task as a dispatching problem instead of simply a pricing problem. We then translate the demand functions along each edge into throughput-reward tradeoffs (i.e., the maximum reward can be achieved along this edge with fixed throughput), and then formulate the problem as an optimization with throughputs (along each edge at edge time slot) as variables.

In our algorithm, we overcome two major difficulties: (i) we first reduce the throughput-reward tradeoff, which in general is not concave, to its convex hull by allowing for randomized pricing schemes (commonly adopted in mechanism design and easy to implement in practice), and (ii) then we solve the MDP with continuous state by formulating it as a convex program. In particular, all the constraints in the convex program are linear.

We emphasize that changing the perspective from pricing to dispatching is critical to enable the global optimization for this problem instead of using local optimization (such as surge pricing) only. Although our approach is quite different from the industry, it can be easily adopted in practice. We discuss such advantages of our approach in details in the full version of this paper [5].

\subsection{Dynamic Environment}

As the environment varies over time in practice, we allow the traveling cost, the valuation and demand of the customers along each edge to be different at each time period. Despite of the high complexity of the dynamics in such an environment, we are able to reduce any general instance to a simplified and restricted instance and the optimal solution of the general instance can be easily recovered from the optimal solution of the simplified instance.

\subsection{Our Contribution}

In this paper, we introduce a simple model for the dispatching problem that can be used for a wide range of general settings (see the full version for detailed discussions on the general settings and how they can be reduced to our simple model [5]). Dynamic environments, as one of the general settings, commonly exist in practice [6], where the prior information (e.g., frequency and values of requests, the traveling costs) may be different for each time period. Such dynamic environments pose additional challenges to the pricing and dispatching problem. To the best of our knowledge, we are the first to incorporate such dynamics into a unified model.

With such a model, we then formalize the problem as a mathematical program with linear constraints by introducing the throughputreward tradeoffs. Although in general the program is not convex, we convexify it by introducing randomized prices and hence the optimal solution can be computed efficiently. One major merit of this approach is that one can easily introduce additional constraints to the problem with few change to the algorithm. For example, in practice, the supply might be asymmetric and correlated over time and the platform may want the distribution of the supply to be stationary in long run.

We also provide a characterization of the optimal solution via primal-dual analysis. In particular, a dynamic pricing scheme is optimal if and only if the marginal contribution of the throughput along each edge equals to the system-wise marginal contribution of additional supply minus the difference of the long term contributions of unit supply at the origin and the destination.

\subsection{Empirical Analysis}

Finally, we perform extensive empirical analysis based on a publicly available dataset with more than 8.5 million orders. We use revenue of the platform as our objective and compare our policy with the fixed pricing policy and the surge pricing policy which is intensively studied in the literature $[3,4,6]$. We perform simulations in both static and dynamic environments.

Our simulation results show that, in the static environment, our optimal pricing scheme outperforms the fixed pricing and surge pricing policies by about $24 \%$ and $17 \%$, respectively. In the dynamic environment, our optimal pricing scheme outperforms the other two policies by about $33 \%, 60 \%$, respectively.

Furthermore, our simulations show that our optimal policy has much stronger ability in dispatching vehicles and balancing the supply and demand across the entire system than other policies, which results directly in its performance boost.

\section{REFERENCES}

[1] Santiago Balseiro, Max Lin, Vahab Mirrokni, Renato Paes Leme, and Song Zuo. 2017. Dynamic revenue sharing. In NIPS 2017.

[2] Siddhartha Banerjee, Carlos Riquelme, and Ramesh Johari. 2015. Pricing in Ride-share Platforms: A Queueing-Theoretic Approach. (2015).

[3] Gerard P Cachon, Kaitlin M Daniels, and Ruben Lobel. 2016. The role of surge pricing on a service platform with self-scheduling capacity. (2016).

[4] Juan Camilo Castillo, Dan Knoepfle, and Glen Weyl. 2017. Surge pricing solves the wild goose chase. In EC'17. ACM, 241-242.

[5] Mengjing Chen, Weiran Shen, Pingzhong Tang, and Song Zuo. 2017. Optimal Vehicle Dispatching Schemes via Dynamic Pricing. arXiv:1707.01625 (2017).

[6] M Keith Chen and Michael Sheldon. 2015. Dynamic pricing in a labor market: Surge pricing and flexible work on the Uber platform. Technical Report.

[7] Judd Cramer and Alan B Krueger. 2016. Disruptive change in the taxi business: The case of Uber. The American Economic Review 106, 5 (2016), 177-182.

[8] Vincent P Crawford and Juanjuan Meng. 2011. New york city cab drivers' labor supply revisited: Reference-dependent preferences with rationalexpectations targets for hours and income. The American Economic Review 101, 5 (2011).

[9] Zhixuan Fang, Longbo Huang, and Adam Wierman. 2017. Prices and subsidies in the sharing economy. In $W W W$ 2017. 53-62.

[10] Michel Gendreau, Alain Hertz, and Gilbert Laporte. 1994. A tabu search heuristic for the vehicle routing problem. Management science 40, 10 (1994), 1276-1290.

[11] Gianpaolo Ghiani, Francesca Guerriero, Gilbert Laporte, and Roberto Musmanno. 2003. Real-time vehicle routing: Solution concepts, algorithms and parallel computing strategies. European fournal of Operational Research 151, 1 (2003).

[12] Peter F Kostiuk. 1990. Compensating differentials for shift work. Journal of political Economy 98, 5, Part 1 (1990), 1054-1075.

[13] Gilbert Laporte. 1992. The vehicle routing problem: An overview of exact and approximate algorithms. European journal of operational research 59, 3 (1992).

[14] R Preston McAfee and Vera Te Velde. 2006. Dynamic pricing in the airline industry. forthcoming in Handbook on Economics and Information Systems, Ed: Tf Hendershott, Elsevier (2006).

[15] Gerald S Oettinger. 1999. An empirical analysis of the daily labor supply of stadium venors. Fournal of political Economy 107, 2 (1999), 360-392.

[16] Joanna Stavins. 2001. Price discrimination in the airline market: The effect of market concentration. Review of Economics and Statistics 83, 1 (2001), 200-202. 